\title{
Theory of rare charm decays into leptons
}

\author{
Alexey A Petrov*† \\ Department of Physics and Astronomy \\ Wayne State University \\ Detroit, MI 48201, USA \\ E-mail: apetrov@wayne.edu
}

Studies of rare decays of charmed mesons into the final states containing leptons is an important vehicle in low-energy searches for new physics. I review theoretical implications of those studies involving transitions with and without lepton flavor conservation. I argue that lepton-flavor violation (LFV) could be successfully studied in heavy quarkonium transitions yielding more sensitive results than similar searches involving $D$ mesons.

9th International Workshop on the CKM Unitarity Triangle

28 November - 3 December 2016

Tata Institute for Fundamental Research (TIFR), Mumbai, India

* Speaker.

${ }^{\dagger}$ This work has been supported in part by the U.S. Department of Energy under contract DE-SC0007983. 


\section{Introduction}

Studies of flavor-changing neutral current (FCNC) transitions with charm quarks is an important vehicle in low-energy searches for new physics (NP) [1]. The primary reason for it is the fact that the standard model (SM) Lagrangian does not contain terms that allow for a change of quark or lepton flavor while conserving their electric charge. Physical processes that represent FCNC transitions are, however, possible in the SM due to quantum fluctuations, i.e. by considering electroweak interactions at one loop. If NP interactions are such that FCNC transitions are possible, either due to elementary interactions in NP Lagrangian or via loop effects with new particles [2], studies of FCNC decays can prove useful in constraining properties of new physics states.

FCNC transition rates are not necessarily small in the SM. Due to the left-handed nature of weak interactions, such currents would be induced with the coefficients that are proportional to the masses (squared) of quarks running in the electroweak loop diagrams generating FCNC transitions. They are expected be small in $D$-decays both due to relatively small mass of the intermediate bottom quark and tiny values of corresponding Cabbibo-Kobayashi-Maskawa (CKM) matrix elements. While some enhancements are possible due to long-distance QCD effects, transitions rates for rare decays such as $D^{0} \rightarrow \ell^{+} \ell^{-}$are still small and have never been observed. This fact makes them a prime target for new physics searches in low energy experiments. Decays of charmed states can probe a variety of NP scenarios due to availability of large datasets of charmed particle decays.

In what follows I shall review the theoretical status of leptonic rare decays of charmed meson states. I shall argue that such decays can probe both quark-flavor violating (QFV) and leptonflavor violating (LFV) transitions. Since both up-quark and lepton FCNC transitions are generally expected to be small, it would be reasonable to consider QFV and LFV transitions separately.

\section{Lepton flavor conserving rare decays}

Lepton-flavor conserving rare decays of $D$ mesons are mediated by quark-level transitions $c \rightarrow$ $u \bar{\ell} \ell$ and $c \rightarrow u \gamma^{*}$ (followed by $\gamma^{*} \rightarrow \bar{\ell} \ell$ ). Due to the hierarchical structure of the CKM matrix, the Glashow-Iliopoulos-Maiani (GIM) mechanism is very effective in these transitions, which makes corresponding SM branching ratios small [1].

In the SM, the short distance contribution to $c \rightarrow u \overline{\ell \ell}$ and $c \rightarrow u \gamma^{*}$ follows from the Lagrangian [1]

$$
\mathscr{L}_{\text {eff }}=\frac{4 G_{F}}{\sqrt{2}} \sum_{i=7,9,10} C_{i} O_{i}
$$

where $G_{F}$ is the Fermi constant and the effective operators are defined as

$$
\begin{aligned}
& O_{7}=-\frac{g_{e m}}{16 \pi^{2}} m_{c}\left(\bar{\ell}_{L} \gamma_{\mu} \ell_{L}\right)\left(\bar{u}_{L} \gamma^{\mu} c_{L}\right), \\
& O_{9}=\frac{\alpha}{4 \pi}\left(\bar{\ell} \gamma_{\mu} \ell\right)\left(\bar{u}_{L} \gamma^{\mu} c_{L}\right), O_{10}=\frac{\alpha}{4 \pi}\left(\bar{\ell} \gamma_{\mu} \gamma_{5} \ell\right)\left(\bar{u}_{L} \gamma^{\mu} c_{L}\right) .
\end{aligned}
$$

where, numerically, the Wilson coefficients are $C_{9}\left(\mu=m_{c}\right)=0.198\left|V_{u b} V_{c b}\right|, C_{10}\left(\mu=m_{c}\right) \simeq 0$ and $C_{7}^{\text {eff }}\left(\mu=m_{c}\right)=-0.0025$ for $m_{c}=1.3 \mathrm{GeV}$ (see [16] for details). 
All possible heavy NP contributions that generate $c \rightarrow u \ell^{+} \ell^{-}$transitions can be summarized in terms of an effective Lagrangian $\mathscr{L}_{\mathrm{NP}}^{\text {rare }}$.

$$
\mathscr{L}_{\mathrm{NP}}^{\text {rare }}=-\sum_{i=1}^{10} \frac{\widetilde{\mathrm{C}}_{i}(\mu)}{\Lambda^{2}} \widetilde{Q}_{i}
$$

where $\widetilde{\mathrm{C}}_{i}$ are the Wilson coefficients, $\widetilde{Q}_{i}$ are the effective operators, and $\Lambda$ represents a scale of possible new physics interactions at which $\widetilde{Q}_{i}$ are generated. There are only ten of those operators of dimension six,

$$
\begin{array}{ll}
\widetilde{Q}_{1}=\left(\bar{\ell}_{L} \gamma_{\mu} \ell_{L}\right)\left(\bar{u}_{L} \gamma^{\mu} c_{L}\right), & \widetilde{Q}_{4}=\left(\bar{\ell}_{R} \ell_{L}\right)\left(\bar{u}_{R} c_{L}\right), \\
\widetilde{Q}_{2}=\left(\bar{\ell}_{L} \gamma_{\mu} \ell_{L}\right)\left(\bar{u}_{R} \gamma^{\mu} c_{R}\right), & \widetilde{Q}_{5}=\left(\bar{\ell}_{R} \sigma_{\mu v} \ell_{L}\right)\left(\bar{u}_{R} \sigma^{\mu v} c_{L}\right), \\
\widetilde{Q}_{3}=\left(\bar{\ell}_{L} \ell_{R}\right)\left(\bar{u}_{R} c_{L}\right), &
\end{array}
$$

where five additional operators $\widetilde{Q}_{6}, \ldots, \widetilde{Q}_{10}$ that can be obtained from operators in Eq. (2.4) by interchanging left- and right-handed fields (i.e. switching $L \leftrightarrow R$ ), e.g. $\widetilde{Q}_{6}=\left(\bar{\ell}_{R} \gamma_{\mu} \ell_{R}\right)\left(\bar{u}_{R} \gamma^{\mu} c_{R}\right)$, $\widetilde{Q}_{7}=(\alpha / 4)\left(\bar{\ell}_{R} \gamma_{\mu} \ell_{R}\right)\left(\bar{u}_{L} \gamma^{\mu} c_{L}\right)$, etc.

The effective Lagrangian of Eq. (2.4) leads to lepton-flavor conserving QFV transitions. Of those, the simplest one is a rare decay $D^{0} \rightarrow \ell^{+} \ell^{-}$. Not all operators from Eq. (2.4) contribute to the decay rate, as some matrix elements (or their linear combinations) vanish in the calculation of $\mathscr{B}\left(D^{0} \rightarrow \ell^{+} \ell^{-}\right)$. For instance, $\left\langle\ell^{+} \ell^{-}\left|\widetilde{Q}_{5}\right| D^{0}\right\rangle=\left\langle\ell^{+} \ell^{-}\left|\widetilde{Q}_{10}\right| D^{0}\right\rangle=0$ (quantum numbers mismatch), $\left\langle\ell^{+} \ell^{-}\left|Q_{9}\right| D^{0}\right\rangle \equiv(\alpha / 4)\left\langle\ell^{+} \ell^{-}\left|\left(\widetilde{Q}_{1}+\widetilde{Q}_{7}\right)\right| D^{0}\right\rangle=0$ (vector current conservation), etc. This transition has a very small SM contribution [1], so it could serve as a clean probe of amplitudes induced by NP particles. The most general $D^{0} \rightarrow \ell^{+} \ell^{-}$decay amplitude is given by

$$
\mathscr{A}\left(D^{0} \rightarrow \ell^{+} \ell^{-}\right)=\bar{u}\left(p_{-}, s_{-}\right)\left[A+\gamma_{5} B\right] v\left(p_{+}, s_{+}\right),
$$

where $\bar{u}\left(p_{-}, s_{-}\right)$and $v\left(p_{+}, s_{+}\right)$are leptons' spinors. The constants $A$ and $B$ depend on the Wilson coefficients of the Lagrangian Eq. (2.3) and some hadronic parameters,

$$
\begin{aligned}
|A| & =\frac{f_{D} M_{D}^{2}}{4 \Lambda^{2} m_{c}}\left[\widetilde{C}_{3-8}+\widetilde{C}_{4-9}\right], \\
|B| & =\frac{f_{D}}{4 \Lambda^{2}}\left[2 m_{\ell}\left(\widetilde{C}_{1-2}+\widetilde{C}_{6-7}\right)+\frac{M_{D}^{2}}{m_{c}}\left(\widetilde{C}_{4-3}+\widetilde{C}_{9-8}\right)\right],
\end{aligned}
$$

with $\widetilde{C}_{i-k} \equiv \widetilde{C}_{i}-\widetilde{C}_{k}$. The amplitude of Eq. (2.5) results in the branching fraction,

$$
\mathscr{B}\left(D^{0} \rightarrow \ell^{+} \ell^{-}\right)=\frac{M_{D}}{8 \pi \Gamma_{\mathrm{D}}} \sqrt{1-\frac{4 m_{\ell}^{2}}{M_{D}^{2}}}\left[\left(1-\frac{4 m_{\ell}^{2}}{M_{D}^{2}}\right)|A|^{2}+|B|^{2}\right],
$$

According to Eq. (2.6), the standard model contribution vanishes in the $m_{\ell} \rightarrow 0$ limit. Any NP model that contribute to $D^{0} \rightarrow \ell^{+} \ell^{-}$can be constrained from the bounds on the Wilson coefficients in Eq. (2.6). It is important to point out that because of the helicity suppression, studies of $\mathscr{B}\left(D^{0} \rightarrow\right.$ $\left.e^{+} e^{-}\right)$vs. $\mathscr{B}\left(D^{0} \rightarrow \mu^{+} \mu^{-}\right)$(and therefore analyses of lepton universality in those decays) are very complicated experimentally (see Sec. 4 for more on this). 
Experimental studies of $D^{0} \rightarrow \ell^{+} \ell^{-}$transitions result in the upper bounds on the branching fractions $[3,4]$,

$$
\mathscr{B}\left(D^{0} \rightarrow \mu^{+} \mu^{-}\right)<7.6 \times 10^{-9}, \mathscr{B}\left(D^{0} \rightarrow e^{+} e^{-}\right)<7.9 \times 10^{-8},
$$

In studying NP contributions to rare decays in charm, it might be advantageous to study correlations of various processes, for instance $D^{0} \bar{D}^{0}$ mixing and rare decays [5].

The effective operators that do not contribute to $D^{0} \rightarrow \ell^{+} \ell^{-}$can be studied in rare semileptonic $[6,7]$ and radiative leptonic $D$-decays. Finally, similar decays with neutrino final states can be used to constrain not only possible NP contributions to $c \rightarrow u v \bar{v}$ [8], but also NP models with light dark matter particles [9], as those transitions have the same experimental signature.

\section{Lepton flavor violating rare decays}

Lepton flavor does not need to be conserved. In the standard model the FCNC interactions in the lepton sector are small, as flavor-violating transition is proportional to square of neutrino masses. This fact makes the rates of LFV FCNC interactions tiny in teh SM. There are, however, many new physics models where lepton flavor is also not conserved. This makes studies of LFV a background-free search for such models of NP.

The discussion of lepton-flavor conserving rare decays in Sect. 2 could be easily extended to include LFV transitions. For example, the rate of LFV leptonic decays of $D$ would be,

$$
\mathscr{B}\left(D^{0} \rightarrow \mu^{+} e^{-}\right)=\frac{M_{D}}{8 \pi \Gamma_{\mathrm{D}}}\left(1-\frac{m_{\mu}^{2}}{M_{D}^{2}}\right)^{2}\left[|A|^{2}+|B|^{2}\right]
$$

where I neglected the electron mass. The constants $A$ and $B$ are defined in Eq. (2.5). Experimental bounds on LFV decays exist [3], e.g.

$$
\mathscr{B}\left(D^{0} \rightarrow \mu^{ \pm} e^{\mp}\right)<1.3 \times 10^{-8} .
$$

It is important to notice that the LFV decay in Eq. (3.1) involves FCNC transitions twice, both on quark and lepton sides of the effective operator that generates such interaction. While this is indeed possible, such operators must be additionally suppressed in many NP models compared to the similar lepton-flavor conserving transitions, say of Eq. (2.7). Thus, if we insist on studying LFV transitions in meson or baryon decays, we should require flavor conservation on the quark side. This immediately implies that Eq. (3.1) and similar ones will only probe the operators of the type $(\bar{\mu} e)(\bar{u} u),(\bar{\mu} e)(\bar{c} c)$, or $(\bar{\mu} e)(\bar{b} b)$. All of those decays are suppressed by small combinations of the CKM factors $V_{c b}^{*} V_{u b}$ resulting from quark FCNCs in the SM in $c \rightarrow u \bar{\mu} e$. It is thus more beneficial to probe those operators in the decays that do not involve quark FCNC interactions, such as two-body $b \bar{b}, c \bar{c}$, or $u \bar{u}$ quarkonium decays [10]. This argument can be easily extended for other LFV transitions of the types $D \rightarrow \bar{\ell}_{1} \ell_{2}$ or $D \rightarrow M \bar{\ell}_{1} \ell_{2}$ where $M$ is a light-quark meson.

The effective Lagrangian describing such transition, $\mathscr{L}_{\text {eff }}$, can then be divided into the dipole part, $\mathscr{L}_{D}$, a part that involves four-fermion interactions, $\mathscr{L}_{\ell q}$, and a gluonic part, $\mathscr{L}_{G}$,

$$
\mathscr{L}_{\text {eff }}=\mathscr{L}_{D}+\mathscr{L}_{\ell q}+\mathscr{L}_{G}+\ldots
$$


Here the ellipses denote effective operators that are not relevant for the following discussion. The dipole part in Eq. (3.3) is usually written as [10,11]

$$
\mathscr{L}_{D}=-\frac{m_{2}}{\Lambda^{2}}\left[\left(C_{D R}^{\ell_{1} \ell_{2}} \bar{\ell}_{1} \sigma^{\mu v} P_{L} \ell_{2}+C_{D L}^{\ell_{1} \ell_{2}} \bar{\ell}_{1} \sigma^{\mu v} P_{R} \ell_{2}\right) F_{\mu v}+\text { h.c. }\right],
$$

where $P_{\mathrm{R}, \mathrm{L}}=\left(1 \pm \gamma_{5}\right) / 2$ is the right (left) chiral projection operator. The Wilson coefficients would, in general, be different for different leptons $\ell_{i}$.

The four-fermion (dimension-six) lepton-quark Lagrangian is given by

$$
\begin{gathered}
\mathscr{L}_{\ell q}=-\frac{1}{\Lambda^{2}} \sum_{q}\left[\left(C_{V R}^{q \ell_{1} \ell_{2}} \bar{\ell}_{1} \gamma^{\mu} P_{R} \ell_{2}+C_{V L}^{q \ell_{1} \ell_{2}} \bar{\ell}_{1} \gamma^{\mu} P_{L} \ell_{2}\right) \bar{q} \gamma_{\mu} q\right. \\
+\left(C_{A R}^{q \ell_{1} \ell_{2}} \bar{\ell}_{1} \gamma^{\mu} P_{R} \ell_{2}+C_{A L}^{q \ell_{1} \ell_{2}} \bar{\ell}_{1} \gamma^{\mu} P_{L} \ell_{2}\right) \bar{q} \gamma_{\mu} \gamma_{5} q \\
+m_{2} m_{q} G_{F}\left(C_{S R}^{q \ell_{1} \ell_{2}} \bar{\ell}_{1} P_{L} \ell_{2}+C_{S L}^{q \ell_{1} \ell_{2}} \bar{\ell}_{1} P_{R} \ell_{2}\right) \bar{q} q \\
+m_{2} m_{q} G_{F}\left(C_{P R}^{q \ell_{1} \ell_{2}} \bar{\ell}_{1} P_{L} \ell_{2}+C_{P L}^{q \ell_{1} \ell_{2}} \bar{\ell}_{1} P_{R} \ell_{2}\right) \bar{q} \gamma_{5} q \\
\left.+m_{2} m_{q} G_{F}\left(C_{T R}^{q \ell_{1} \ell_{2}} \bar{\ell}_{1} \sigma^{\mu v} P_{L} \ell_{2}+C_{T L}^{q \ell_{1} \ell_{2}} \bar{\ell}_{1} \sigma^{\mu v} P_{R} \ell_{2}\right) \bar{q} \sigma_{\mu v} q+\text { h.c. }\right] .
\end{gathered}
$$

The dimension seven gluonic operators can be either generated by some high scale physics or by integrating out heavy quark degrees of freedom [11, 12],

$$
\begin{aligned}
\mathscr{L}_{G}=-\frac{m_{2} G_{F}}{\Lambda^{2}} \frac{\beta_{L}}{4 \alpha_{s}}[ & {\left[\left(C_{G R}^{\ell_{1} \ell_{2}} \bar{\ell}_{1} P_{L} \ell_{2}+C_{G L}^{\ell_{1} \ell_{2}} \bar{\ell}_{1} P_{R} \ell_{2}\right) G_{\mu v}^{a} G^{a \mu v}\right.} \\
+ & \left.\left(C_{\overline{G R}}^{\ell_{1} \ell_{2}} \bar{\ell}_{1} P_{L} \ell_{2}+C_{\bar{G} L}^{\ell_{1} \ell_{2}} \bar{\ell}_{1} P_{R} \ell_{2}\right) G_{\mu v}^{a} \widetilde{G}^{a \mu v}+\text { h.c. }\right] .
\end{aligned}
$$

Here $\beta_{L}=-9 \alpha_{s}^{2} /(2 \pi)$ is defined for the number of light active flavors, $L$, relevant to the scale of the process. All Wilson coefficients should also be calculated at the same scale. $\widetilde{G}^{a \mu v}=$ $(1 / 2) \varepsilon^{\mu \nu \alpha \beta} G_{\alpha \beta}^{a}$ is a dual to the gluon field strength tensor.

The most general expression for the $V \rightarrow \ell_{1} \bar{\ell}_{2}$ decay amplitude can be written as

$$
\begin{aligned}
\mathscr{A}\left(V \rightarrow \ell_{1} \bar{\ell}_{2}\right)=\bar{u}\left(p_{1}, s_{1}\right)\left[A_{V}^{\ell_{1} \ell_{2}} \gamma_{\mu}\right. & +B_{V}^{\ell_{1} \ell_{2}} \gamma_{\mu} \gamma_{5}+\frac{C_{V}^{\ell_{1} \ell_{2}}}{m_{V}}\left(p_{2}-p_{1}\right)_{\mu} \\
& \left.+\frac{i D_{V}^{\ell_{1} \ell_{2}}}{m_{V}}\left(p_{2}-p_{1}\right)_{\mu} \gamma_{5}\right] v\left(p_{2}, s_{2}\right) \varepsilon^{\mu}(p) .
\end{aligned}
$$

where $A_{V}^{\ell_{1} \ell_{2}}, B_{V}^{\ell_{1} \ell_{2}}, C_{V}^{\ell_{1} \ell_{2}}$, and $D_{V}^{\ell_{1} \ell_{2}}$ are constants which depend on Wilson coefficients of the effective Lagrangian of Eq. (3.3) as well as on hadronic effects associated with meson-to-vacuum matrix elements (decay constants). The exact form of these form-factors is rather cumbersome and can be found in [10]. The amplitude of Eq. (3.7) leads to the branching fraction, which is convenient to represent as

$$
\begin{aligned}
\frac{\mathscr{B}\left(V \rightarrow \ell_{1} \bar{\ell}_{2}\right)}{\mathscr{B}\left(V \rightarrow e^{+} e^{-}\right)} & =\left(\frac{m_{V}\left(1-y^{2}\right)}{4 \pi \alpha f_{V} Q_{q}}\right)^{2}\left[\left(\left|A_{V}^{\ell_{1} \ell_{2}}\right|^{2}+\left|B_{V}^{\ell_{1} \ell_{2}}\right|^{2}\right)+\frac{1}{2}\left(1-2 y^{2}\right)\left(\left|C_{V}^{\ell_{1} \ell_{2}}\right|^{2}+\left|D_{V}^{\ell_{1} \ell_{2}}\right|^{2}\right)\right. \\
& \left.+y \operatorname{Re}\left(A_{V}^{\ell_{1} \ell_{2}} C_{V}^{\ell_{1} \ell_{2} *}+i B_{V}^{\ell_{1} \ell_{2}} D_{V}^{\ell_{1} \ell_{2} *}\right)\right] .
\end{aligned}
$$




\begin{tabular}{ccccccc} 
& Leptons & \multicolumn{5}{c}{ Initial state (quark) } \\
\hline Wilson coef, $\mathrm{GeV}^{-2}$ & $\ell_{1} \ell_{2}$ & $\Upsilon(1 S)(b)$ & $\Upsilon(2 S)(b)$ & $\Upsilon(3 S)(b)$ & $J / \psi(c)$ & $\phi(s)$ \\
\hline & $\mu \tau$ & $5.6 \times 10^{-6}$ & $4.1 \times 10^{-6}$ & $3.5 \times 10^{-6}$ & $5.5 \times 10^{-5}$ & $\mathrm{n} / \mathrm{a}$ \\
$\left|C_{V L}^{q \ell_{1} \ell_{2}} / \Lambda^{2}\right|$ & $e \tau$ & - & $4.1 \times 10^{-6}$ & $4.1 \times 10^{-6}$ & $1.1 \times 10^{-4}$ & $\mathrm{n} / \mathrm{a}$ \\
& $e \mu$ & - & - & - & $1.0 \times 10^{-5}$ & $2 \times 10^{-3}$ \\
\hline \multirow{2}{*}{$\left|C_{V R}^{q \ell_{1} \ell_{2}} / \Lambda^{2}\right|$} & $\mu \tau$ & $5.6 \times 10^{-6}$ & $4.1 \times 10^{-6}$ & $3.5 \times 10^{-6}$ & $5.5 \times 10^{-5}$ & $\mathrm{n} / \mathrm{a}$ \\
& $e \tau$ & - & $4.1 \times 10^{-6}$ & $4.1 \times 10^{-6}$ & $1.1 \times 10^{-4}$ & $\mathrm{n} / \mathrm{a}$ \\
& $e \mu$ & - & - & - & $1.0 \times 10^{-5}$ & $2 \times 10^{-3}$ \\
\hline & $\mu \tau$ & $4.4 \times 10^{-2}$ & $3.2 \times 10^{-2}$ & $2.8 \times 10^{-2}$ & 1.2 & $\mathrm{n} / \mathrm{a}$ \\
$\left|C_{T L}^{q \ell_{1} \ell_{2}} / \Lambda^{2}\right|$ & $e \tau$ & - & $3.3 \times 10^{-2}$ & $3.2 \times 10^{-2}$ & 2.4 & $\mathrm{n} / \mathrm{a}$ \\
& $e \mu$ & - & - & - & 4.8 & $1 \times 10^{4}$ \\
\hline \multirow{2}{*}{$\left|C_{T R}^{q \ell_{1} \ell_{2}} / \Lambda^{2}\right|$} & $\mu \tau$ & $4.4 \times 10^{-2}$ & $3.2 \times 10^{-2}$ & $2.8 \times 10^{-2}$ & 1.2 & $\mathrm{n} / \mathrm{a}$ \\
& $e \tau$ & - & $3.3 \times 10^{-2}$ & $3.2 \times 10^{-2}$ & 2.4 & $\mathrm{n} / \mathrm{a}$ \\
& $e \mu$ & - & - & - & 4.8 & $1 \times 10^{4}$ \\
\hline
\end{tabular}

Table 1: Constraints on the Wilson coefficients of four-fermion operators from $1^{--}$quarkonium decays. Dashes signify the absence of experimental data; "n/a" means that the transition is forbidden by phase space.

Here $y=m_{\ell_{2}} / m_{V}$. Comparing Eq. (3.8) to experimental data one can constrain the Wilson coefficients of Eq. (3.5) that correspond to vector and tensor operators. They can be found in Table 1 $[10,13]$.

Similar analysis can be performed for LFV decays of scalar $(S)$ and pseudoscalar $(P)$ quarkonium states. The most general expression for the $S / P \rightarrow \ell_{1} \bar{\ell}_{2}$ decay amplitude is

$$
\mathscr{A}\left(S / P \rightarrow \ell_{1} \bar{\ell}_{2}\right)=\bar{u}\left(p_{1}, s_{1}\right)\left[E_{S / P}^{\ell_{1} \ell_{2}}+i F_{S / P}^{\ell_{1} \ell_{2}} \gamma_{5}\right] v\left(p_{2}, s_{2}\right)
$$

where, as in the case of the vector quarkonium decays $E_{S / P}^{\ell_{1} \ell_{2}}$ and $F_{S / P}^{\ell_{1} \ell_{2}}$ are dimensionless constants which depend on the underlying Wilson coefficients of the effective Lagrangian of Eq. (3.3) and on decay constants.

The amplitude of Eq. (3.9) leads to the branching ratio for flavor off-diagonal leptonic decays of pseudoscalar mesons:

$$
\mathscr{B}\left(S / P \rightarrow \ell_{1} \bar{\ell}_{2}\right)=\frac{m_{S / P}}{8 \pi \Gamma_{S / P}}\left(1-y^{2}\right)^{2}\left[\left|E_{S / P}^{\ell_{1} \ell_{2}}\right|^{2}+\left|F_{S / P}^{\ell_{1} \ell_{2}}\right|^{2}\right] .
$$

Here $\Gamma_{S / P}$ is the total width of the scalar or pseudoscalar state and $y=m_{\ell_{2}} / m_{S / P}$. It is interesting to note that scalar quarkonium decays are mostly sensitive to the scalar operators in Eq. (3.5), eliminating the need an assumption of single operator dominance [10].

The decays of the scalar $S=\chi_{q 0}$ or pseudoscalar $P=\eta_{q}$ states are difficult to study at colliders. However, the following trick could be employed. Since vector states are abundantly produced both at $e^{+} e^{-}$and hadronic machines, a resonant two-body radiative transitions of vector states

$$
\mathscr{B}\left(V \rightarrow \gamma \ell_{1} \bar{\ell}_{2}\right)=\mathscr{B}(V \rightarrow \gamma S / P) \mathscr{B}\left(S / P \rightarrow \ell_{1} \bar{\ell}_{2}\right),
$$




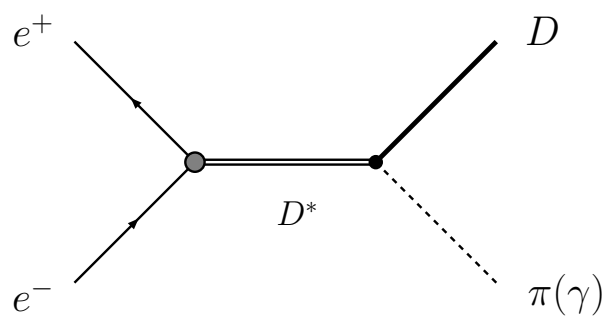

Figure 1: Probing the $c \bar{u} \rightarrow e^{+} e^{-}$vertex with the $D^{*}(2007)^{0}$ resonance production in $e^{+} e^{-}$collisions.

could be used to produce scalar $S$ and/or pseudoscalar $P$ states. The branching ratios for the radiative transitions $V \rightarrow \gamma S / P$ are rather large, e.g.

$$
\begin{aligned}
& \mathscr{B}\left(\psi(2 S) \rightarrow \gamma \chi_{c 0}(1 P)\right)=9.99 \pm 0.27 \% \\
& \mathscr{B}\left(\Upsilon(3 S) \rightarrow \gamma \chi_{b 0}(2 P)\right)=5.9 \pm 0.6 \% \\
& \mathscr{B}\left(J / \psi \rightarrow \gamma \eta_{c}\right)=1.7 \pm 0.4 \%
\end{aligned}
$$

An estimate [14] shows that with the integrated luminosity of $\mathscr{L}=250 \mathrm{fb}^{-1}$ the number of produced $\chi_{b}$ states could reach tens of millions, making such studies very feasible.

\section{Probing rare leptonic charm transitions in production experiments}

As was mentioned in Sect. 2, the rate of the simplest FCNC decay, $D^{0} \rightarrow \ell^{+} \ell^{-}$, is suppressed by the helicity, i.e. by the mass of the final state leptons. This could complicate studies of lepton flavor universality as the branching ratio of $D^{0} \rightarrow e^{+} e^{-}$is tiny. Experimental studies of a similar transition, $D^{*} \rightarrow e^{+} e^{-}$, that is not helicity suppressed, are not feasible as $D^{*}$, contrary to the $D^{0}$, also decays strongly or electromagnetically with much larger rates.

An interesting alternative to studies of $D^{*}$ decays is to measure the corresponding production process $e^{+} e^{-} \rightarrow D^{*}$, as shown in Fig. 1 [15]. This is possible at an $e^{+} e^{-}$collider, such as BEPCII or VEPP-2000, tuned to run at the center-of-mass energy corresponding to the mass of the $D^{*}$ meson, $\sqrt{s} \approx 2007 \mathrm{MeV}$. The produced $D^{* 0}$ resonance, tagged by a single charmed particle in the final state, will decay strongly $\left(D^{* 0} \rightarrow D^{0} \pi^{0}\right)$ or electromagnetically $\left(D^{* 0} \rightarrow D^{0} \gamma\right)$ with branching fractions of $(61.9 \pm 2.9) \%$ and $(38.1 \pm 2.9) \%$ respectively. This process, albeit very rare, has clear advantages for NP studies compared to the $D^{0} \rightarrow e^{+} e^{-}$decay: the helicity suppression is absent, and a richer set of effective operators can be probed. It is also interesting to note that contrary to other rare decays of charmed mesons, long-distance SM contributions are under theoretical control and contribute at the same order of magnitude as the short-distance ones. Similar opportunities exist for $B$-decays as well $[15,17]$.

\section{Conclusions}

The apparent absence of any hints of new particles from current direct searches at Large Hadron Collider (LHC) experiments makes careful studies of their possible quantum effects an 
important tool in our arsenal of methods for probing physics beyond the SM. Abundance of charm data in the current and future low energy flavor experiments makes it possible to study NP in QFV and LFV rare decays of charmed mesons with ever increased precision. The obtained constraints from a variety of methods described in this talk are competitive with the bounds obtained from the continuing direct searches for NP particles at the LHC.

\section{References}

[1] S. Fajfer, arXiv:1509.01997 [hep-ph]; M. Artuso, B. Meadows and A. A. Petrov, Ann. Rev. Nucl. Part. Sci. 58, 249 (2008); G. Burdman and I. Shipsey, Ann. Rev. Nucl. Part. Sci. 53, 431 (2003)

[2] A. A. Petrov and A. E. Blechman, "Effective Field Theories," ISBN: 978-981-4434-92-8, World Scientific Publishing, 2016.

[3] Y. Amhis et al. [Heavy Flavor Averaging Group (HFAG) Collaboration], arXiv:1612.07233 [hep-ex].

[4] R. Aaij et al. [LHCb Collaboration], Phys. Lett. B 725, 15 (2013); R. Aaij et al. Phys. Lett. B 754, 167 (2016); M. Petric et al. [Belle Collaboration], Phys. Rev. D 81, 091102 (2010)

[5] E. Golowich, J. Hewett, S. Pakvasa and A. A. Petrov, Phys. Rev. D 79, 114030 (2009)

[6] S. de Boer and G. Hiller, Phys. Rev. D 93, no. 7, 074001 (2016)

[7] S. Fajfer, N. Kosnik and S. Prelovsek, Phys. Rev. D 76, 074010 (2007); S. Fajfer and N. Kosnik, Eur. Phys. J. C 75, 567 (2015); A. Paul, I. I. Bigi and S. Recksiegel, Phys. Rev. D 83, 114006 (2011)

[8] G. Burdman, E. Golowich, J. L. Hewett and S. Pakvasa, Phys. Rev. D 66, 014009 (2002)

[9] A. Badin and A. A. Petrov, Phys. Rev. D 82, 034005 (2010); Y. G. Aditya, K. J. Healey, A. A. Petrov, Phys. Lett. B 710, 118 (2012); D. Atwood and A. A. Petrov, Phys. Rev. D 71, 054032 (2005)

[10] D. E. Hazard and A. A. Petrov, Phys. Rev. D 94, no. 7, 074023 (2016)

[11] A. Celis, V. Cirigliano and E. Passemar, Phys. Rev. D 89, no. 9, 095014 (2014)

[12] A. A. Petrov and D. V. Zhuridov, Phys. Rev. D 89, no. 3, 033005 (2014)

[13] A. Abada, D. Becirevic, M. Lucente and O. Sumensari, Phys. Rev. D 91, no. 11, 113013 (2015)

H. K. Dreiner, G. Polesello and M. Thormeier, Phys. Rev. D 65, 115006 (2002)

[14] S. Godfrey and H. E. Logan, Phys. Rev. D 93, no. 5, 055014 (2016)

[15] A. Khodjamirian, T. Mannel and A. A. Petrov, JHEP 1511, 142 (2015)

[16] S. de Boer, B. Muller and D. Seidel, JHEP 1608, 091 (2016)

[17] B. Grinstein and J. M. Camalich, Phys. Rev. Lett. 116, no. 14, 141801 (2016) 